\title{
Near-field probing of strong light- matter coupling in single IR antennae
}

Mitrofanov, O., Wang, C.-F., Habteyes, T., Luk, T. S., Klem, J., et al.

O. Mitrofanov, C.-F. Wang, T. G. Habteyes, T. S. Luk, J. F. Klem, I. Brener, H.T. Chen, "Near-field probing of strong light-matter coupling in single IR antennae," Proc. SPIE 11499, Terahertz Emitters, Receivers, and Applications XI, 114990A (20 August 2020); doi: 10.1117/12.2568033

SPIE. Event: SPIE Optical Engineering + Applications, 2020, Online Only 


\title{
Near-field probing of strong light-matter coupling in single IR antennae
}

\author{
O. Mitrofanov, ${ }^{1,2, *}$ C.-F. Wang, ${ }^{3,4,}{ }^{* *}$ T. G. Habteyes, ${ }^{4}$ \\ T. S. Luk, ${ }^{2}$ J. F. Klem, ${ }^{5}$ I. Brener, ${ }^{2}$ and H.-T. Chen. ${ }^{3}$ \\ ${ }^{1}$ Electronic and Electrical Engineering, University College London, London, WC1E 7JE, UK \\ ${ }^{2}$ Center for Integrated Nanotechnologies, Sandia National Labs, Albuquerque, NM 87123 USA \\ ${ }^{3}$ Center for Integrated Nanotechnologies, Los Alamos National Lab., Los Alamos, NM 87545, USA \\ ${ }^{4}$ Center for High Technology Materials, University of New Mexico, Albuquerque, NM 87106, USA \\ 5 Sandia National Laboratories, Albuquerque, NM 87185, USA
}

\begin{abstract}
Quantum well intersubband polaritons are traditionally studied in large scale systems, over many wavelengths in size. In this presentation, we demonstrate that it is possible to detect and investigate intersubband polaritons in a single subwavelength nanoantenna in the IR frequency range. We observe polariton formation using a scattering-type near-field microscope and nano-FTIR spectroscopy. We will discuss near-field spectroscopic signatures of plasmonic antennae with and without coupling to the intersubband transition in quantum wells located underneath the antenna. Evanescent field amplitude spectra recorded on the antenna surface show a mode anti-crossing behavior in the strong coupling case. We also observe a corresponding strong-coupling signature in the phase of the detected field. We anticipate that this near-field approach will enable explorations of strong and ultrastrong light-matter coupling in the single nanoantenna regime, including investigations of the elusive effect of ISB polariton condensation.
\end{abstract}

Keywords: polariton, strong light-matter coupling, intersubband transition, quantum well, near-field microscopy, nanoFTIR, plasmonic nanoantenna, dipole resonance.

\section{INTRODUCTION}

The intersubband (ISB) transition is one of the fundamental quantum effects experienced by electrons confined in a quantum well [1]. In addition to the scientific significance, the quantum well systems with ISB transitions found a range of practical applications in optoelectronics and enabled IR detectors and quantum cascade lasers (QCL). When the ISB transition interacts with a cavity or a resonator, the coupling between the resonant photons and electrons in the quantum well can lead to formation of a polariton state, a superposition of the photon and quantum well excitation. In its turn, this light-matter interaction can lead to more exotic quantum phenomena, such as polariton condensation and lasing $[1,2]$.

To limit the impact of inhomogeneities and to maintain coherence over the entire system, it is desired to investigate polariton quantum phenomena in a small (sub-wavelength size) isolated system, for instance in a single nanoscale antenna. However, such a configuration presents a challenging experimental problem in the IR and the terahertz (THz) parts of the spectrum. In this presentation, we will discuss one solution to this problem.

\subsection{Nano-antennas for investigations of ISB polaritons}

In general, a nano-antenna provides a convenient approach to enhance light-matter interaction. It is especially true for ISB excitations in quantum wells, because the optical transition selection rule requires that the electric field vector of light is perpendicular to the quantum well plane. In the past, special microcavities were fabricated to study ISB polaritons. The photon field in such microcavities however had poor field confinement, and light coupling to the cavity was difficult.

* email: o.mitrofanov@ucl.ac.uk

** Present address: Physical Sciences Division, Pacific Northwest National Laboratory, Richland, WA 99352.

Terahertz Emitters, Receivers, and Applications XI, edited by Manijeh Razeghi,

Alexei N. Baranov, Proc. of SPIE Vol. 11499, 114990A - (C) 2020 SPIE

CCC code: $0277-786 \mathrm{X} / 20 / \$ 21 \cdot$ doi: $10.1117 / 12.2568033$ 
In contrast, a small metallic patch or a nanoantenna fabricated on the surface of a quantum well sample naturally provides the required electric field polarization [3]. In addition, a plasmonic nanoantenna can provide strong confinement and resonant enhancement of the optical field, leading to stronger light-matter interaction.

A nanoantenna however has a drawback: a subwavelength size antenna placed on a high-index material with the quantum wells results in very small total scattering cross-section. That makes observing ISB polaritons in a single nanoantenna difficult, and so far, such studies have been performed using arrays of antennae.

Attempts have been made to reach the single-antenna regime by gradually reducing the total number of array elements, and by increasing the separation between antennae in the array [4,5]. However, neither of these approaches reached the single nanoantenna regime, because the signal became too weak to be detected.

Near-field microscopy offers an alternative approach to the problem of single-antenna investigation. It has been shown that a subwavelength antenna yields sufficiently strong signal when studied by a near-field microscope [6-9], for example the scattering type microscope $[10,11]$. Therefore, the question that we need to address is whether a nanoantenna displays an ISB polariton signature in the near-field microscopy signal, and if so, what is the physical mechanism behind the ISB polariton signature.

\subsection{Near-field probing of nano-antenna coupled to the ISB transition}

Figure 1 illustrates the near-field microscopy approach for probing ISB polaritons in a single nanoantenna. A metallic antenna is defined lithographically on the surface of a quantum well sample, and it is excited with a broadband IR pulse. If the excitation is in resonance with the antenna, we expect that resonant plasmonic fields are formed on the antenna surface. To detect these field, we approach the antenna with an AFM probe, which scatters some of the field energy from the antenna surface to a detector positioned in the far-field region.

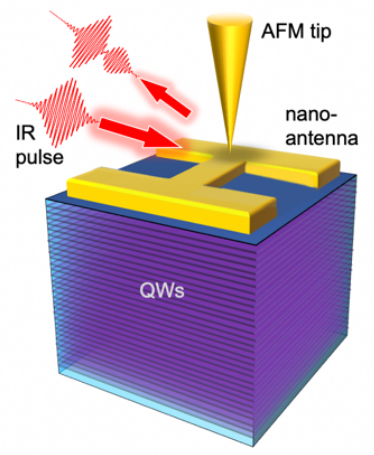

Figure 1. Schematic diagram of near-field probing of ISB polaritons in the single-nanoantenna regime.

For our experiments, we chose two quantum well systems with the ISB transition wavelengths in the region of 10$12 \mu \mathrm{m}$. Each quantum well is made of In $0.53 \mathrm{Ga} 0.47 \mathrm{As}$ with $\mathrm{Al}_{0.48} \mathrm{In}_{0.52} \mathrm{As}$ barriers, and the samples have stacks of 20 quantum wells. We used two samples: one with the quantum well thickness of $9.5 \mathrm{~nm}$; and the second with wider quantum wells, $12.5 \mathrm{~nm}$. The quantum well width allows us to control the electron transition energy from the ground to the excited state, and for the $12.5 \mathrm{~nm}$ quantum well the transition is at $800 \mathrm{~cm}^{-1}$, whereas the transition is at $1150 \mathrm{~cm}^{-1}$ for the $9.5 \mathrm{~nm}$ quantum well [12]. The former will be used as reference.

For the nanoantenna we choose the dogbone geometry (Fig. 1). Arrays of such antennas fabricated on similar quantum well systems showed formation of polaritons in earlier studies, with clear splitting of the ISB transition into two polariton states. This antenna design is also subwavelength: an antenna of about $1 \mu \mathrm{m}$ in size has a dipolar resonance at a wavelength of $\sim 10 \mu \mathrm{m}$. Numerical simulations of this design confirm that the electric field magnitude $|\mathrm{E}|$ for such an antenna is distributed in the region of quantum wells, with a strong normal component satisfying the ISB transition selection rule [12]. Therefore, this antenna design is good for ISB polariton studies: it provides strong subwavelength confinement, which we need in order to achieve the regime of strong coupling. 
The linewidth of the antenna resonance is approximately $200 \mathrm{~cm}^{-1}$ [12]. Therefore, for an antenna with the resonance centered at $1150 \mathrm{~cm}^{-1}$, we expect it to couple to the ISB transition of the $9.5 \mathrm{~nm}$ quantum well sample, but not to the ISB transition in the $12.5 \mathrm{~nm}$ quantum well reference sample.

\section{EXPERIMENTAL RESULTS}

\subsection{Resonant fields on the nano-antenna surface}

We first analyze the dipolar mode of the antenna using the scattering-type near-field microscope. One way to confirm that the mode is supported by the antenna is through analysis of spatial distribution of the fields on the antenna surface. In this experiment we use $\mathrm{CW}$ radiation at the wavelength of $10 \mu \mathrm{m}$ from a mid-IR quantum cascade laser (QCL) and we map the amplitude of the scattered field on the sample surface.

Figure 2 displays the detected amplitude of the scattered field for two nanoantennae: one is selected to be in resonance with the QCL excitation, and the other is selected to have its resonance at a sufficiently different wavelength. To avoid ISB excitation, we use the $12.5 \mathrm{~nm}$ quantum well sample, so that the ISB transition is not activated either.

The near-field maps in Fig. 2 clearly show the outline of the metallic structure of the antenna. In addition, the top map shows that the amplitude on the field is different on the opposite sides of the resonant antenna, indicating formation of the dipolar resonance. For the larger antenna the incident field does not excite the antenna resonance (bottom map), and we find that the scattered field is uniform over the entire metallic surface.

These examples highlight the fact that the near-field microscope provides two different components in the detected signal. In addition to the resonant fields on the surface, the microscope also shows material contrast: metallic features appear brighter than the semiconductor surface. Quantitatively, the scattered field signal from the metallic features of the non-resonant antenna is about twice as strong as the signal from the semiconductor. In an excited antenna, resonant fields are superimposed on the material contrast signal. As a result, the field amplitude changes from low to high going from the front to the back side of the antenna. This corresponds to a superposition of the enhanced scattered field from the metallic surface and the dipolar distribution due to the resonance excitation on the antenna.

We note that the resonant fields appear on the antenna surface only if its resonant frequency matches the incident field frequency. Although this mode is primarily distributed in the semiconductor, the mode energy leaks on the antenna surface by means of surface waves, enabling detection of the mode by the near-field microscope.

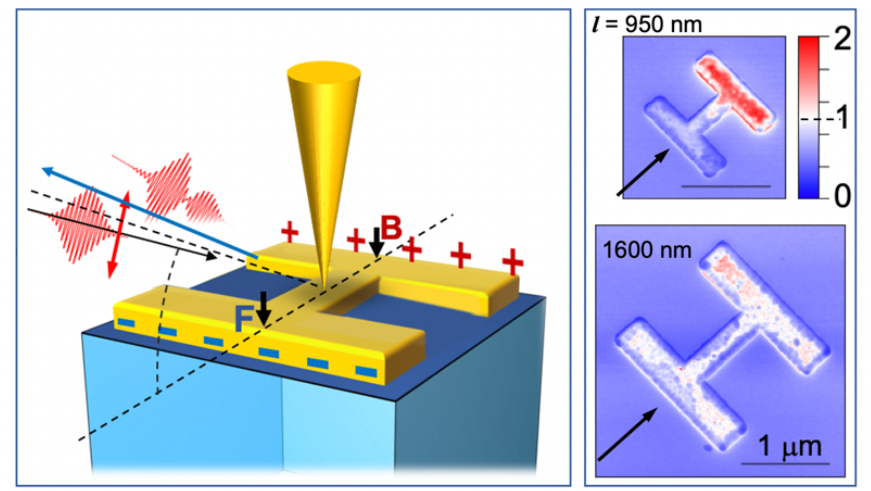

Figure 2. Schematic diagram of antenna excitation (left panel). Near-field amplitude maps in the CW mode for two antennae (right panel). The top map shows an antenna in resonance with the QCL excitation; the bottom map shows a larger antenna with the dipolar resonance at sufficiently lower frequency (adapted from Ref. 12). 


\subsection{Nano-FTIR spectroscopy of nanoantenna}

Now we spectroscopically analyze the evanescent fields on the antenna surface under broadband pulsed excitation covering a spectral region from $800 \mathrm{~cm}^{-1}$ to $1350 \mathrm{~cm}^{-1}$. We detect near-field signal at the bars of the antenna where the resonant field amplitude is the strongest (marked by letters ' $\mathrm{F}$ ' and ' $\mathrm{B}$ ' in Fig. 2). When we compare the Fourier amplitude and phase of the detected field at the two locations, at the front bar and the back bar of the antenna, we find that the FTIR spectrum shows a dip at the resonance frequency of the antenna, $\sim 1100 \mathrm{~cm}^{-1}$, whereas the spectrum of the back bar shows a peak around the same frequency. This is consistent with our interpretation of the near-field signal, i.e. that the signal is superposition of the field scattered by the metallic surface and evanescent resonant field. Depending on the phase of the resonant field with respect to the field scattered by the metallic surface, we see either constructive of destructive interference at the resonance frequency. Therefore, we can spectroscopically analyze properties of this dipolar mode in the antenna by probing the evanescent field on the antenna surface.

Furthermore, we can take advantage of the FTIR spectroscopy capability to extract phase of the detected field, and utilize the phase information in our analysis. In Fig. 3 we plot the scattered signal in the complex plane, using a vector with the length equal to the field amplitude and its direction defined by the phase. The signal vector describes a circular trace in the complex plane as we sweep the Fourier frequency. For the front bar the trace is shown by the red dots and for the back bar, by the blue dots. This phasor representation helps to reveal the two contributions to the near-field signal: the resonant antenna field contribution is a rotating vector, which changes its direction with frequency near the nanoantenna resonance, whereas the frequency-independent contribution is a stationary vector directed to the region where the two circles 'touch'. The phasor diagram in Fig. 3(b) serves as a reference near-field signature of the antenna resonance.
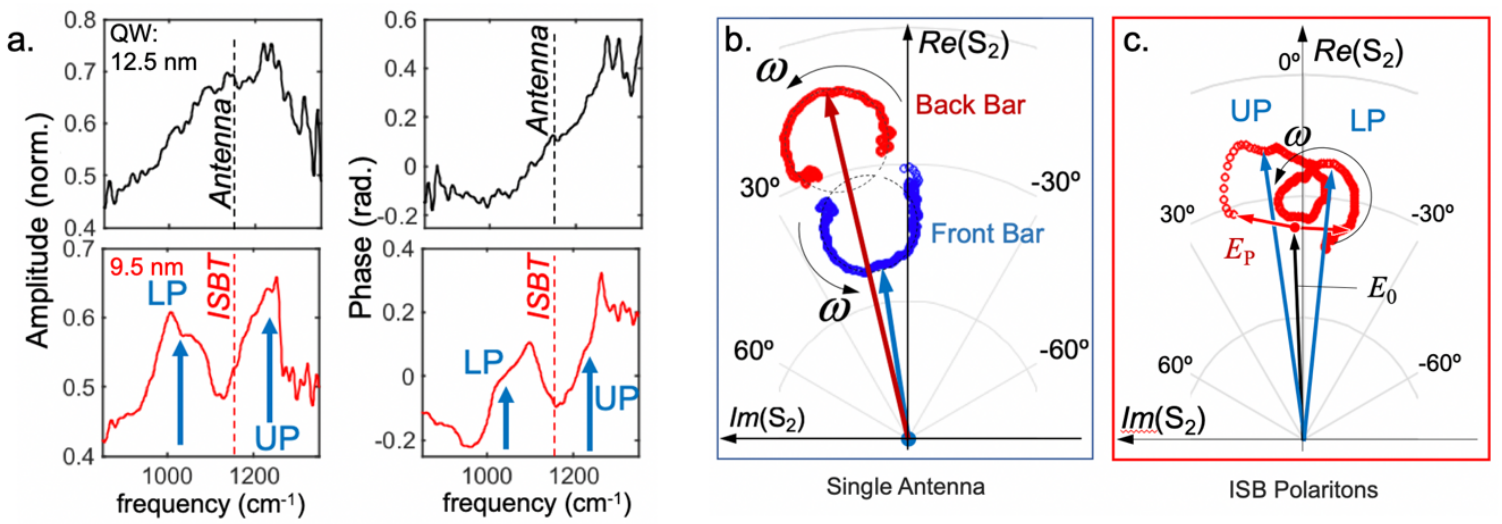

Figure 3. (a) Nano-FTIR spectroscopy analysis on the back bar of the $l=850 \mathrm{~nm}$ antenna. The top row shows the amplitude and phase of the scattered signal for the antenna placed on the stack of the $12.5 \mathrm{~nm}$ wide quantum wells; the bottom row shows the FTIR signal for the antenna on the $9.5 \mathrm{~nm}$ wide quantum wells. (b) Phasor representation of the amplitude and phase information for the antenna on the $12.5 \mathrm{~nm}$ quantum well sample, collected at the back and the front bar of the antenna. (c) Phasor plot for the antenna on the $9.5 \mathrm{~nm}$ quantum well sample (collected at the back bar) showing a frequency trace with two loops, one for each of the polariton branches. Adapted from Ref 12.

\subsection{Near-field signature of ISB polaritons}

Having identified this near-field signatures of the nanoantenna resonance in amplitude and phase spectra, we now introduce coupling to the ISB transition. We select a nanoantenna to match the quantum well excitation for the $9.5 \mathrm{~nm}$ wide well sample and record nano-FTIR amplitude and phase spectra.

As we showed in Fig. 3(a), for the $12.5 \mathrm{~nm}$ quantum well, the nanoantenna does not couple to the ISB transition since it is off resonance, and the nano-FTIR spectrum shows only the antenna resonance signature at $\sim 1150 \mathrm{~cm}^{-1}$. In contrast, the same nanoantenna on the $9.5 \mathrm{~nm}$ sample shows now two distinct peaks in the amplitude spectrum, above and below 
the ISB transition energy, whereas the field enhancement at the resonance frequency of the uncoupled nanoantenna disappears entirely.

This splitting of the nanoantenna peak indicates polariton formation. One peak corresponds to the lower polariton branch and the other corresponds to the upper polariton branch. We also observe a different signature in the phase spectrum, which shows two steps, instead of one, each centered at one of the polariton frequencies. To our knowledge, this is the first observation of ISB polaritons in the single antenna regime. The polaritons show also a distinctly different trace in the complex plane. The phasor now describes a frequency trace with two complete loops indicating that the corresponding phase changes by $\pi$ for each of the two polariton states.

\subsection{ISB Polariton dispersion in single nano-antenna regime}

We now can use this nano-FTIR technique and map dispersion characteristics of the ISB polaritons in the single nanoantenna regime. We fabricate a set of nanoantennae with sizes ranging from 600 to $1600 \mathrm{~nm}$ on two samples with different widths of the quantum wells and, as before, we record amplitude and phase spectra at the same point for every antenna. The results are shown in the maps in Fig. 5, where we combined the amplitude spectra for all the antennae along the horizonal coordinate.

For the $12.5 \mathrm{~nm}$ quantum wells, where the ISB transition is not excited by the incident pulse, only the antenna resonance is present in the map: the amplitude peak shifts in frequency with the inverse antenna length practically linearly. The same nanoantenna set fabricated on the $9.5 \mathrm{~nm}$ QW stack shows a different behavior. In the low-frequency range, it follows the antenna dispersion; however, in the region of the ISB transition, the peak starts shifting to right side of the map and disappears. For antennae with resonance frequencies close to the ISB transition, we see two peaks with a splitting of about $200 \mathrm{meV}$ [12].

This dispersion map shows the polariton anti-crossing behavior in the single nano-antenna regime. We note that all of the data are collected from one sample with a set of 16 antennas of different sizes (Fig. 4a). It demonstrates the power of the nano-FTIR approach. We anticipate that this technique will open doors for investigations of light-matter interaction in the single subwavelength nanoantenna regime.

a.

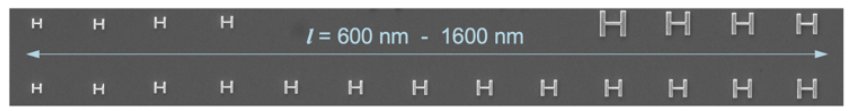

b.
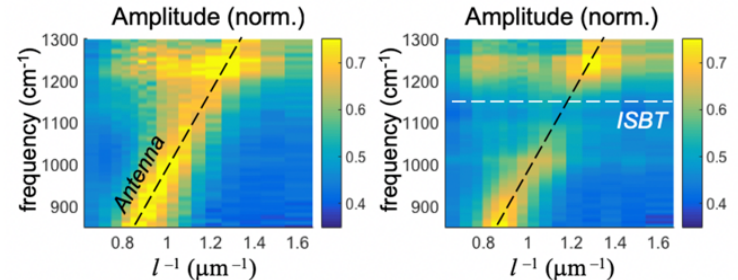

C.

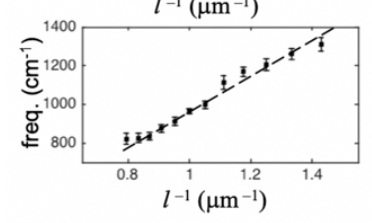

QW: $12.5 \mathrm{~nm}$

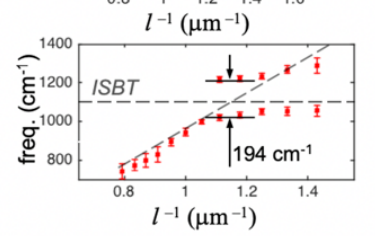

QW: $9.5 \mathrm{~nm}$

Figure 4. (a) SEM image of a set of dogbone antennae used for polariton dispersion mapping. The antennae are spaced $4 \mu \mathrm{m}$ apart. (b) Amplitude spectra for all of the antennae are arranged as maps where the vertical axis represents the frequency and the horizontal axis represents the inverse antenna size. The left map shows spectra for the antennae on the $12.5 \mathrm{~nm}$ quantum well sample, and the right map shows spectra for antennae on the $9.5 \mathrm{~nm}$ quantum well sample. (c) Peak frequencies extracted from the amplitude maps for varying inverse antenna size. Adapted from Ref. 12. 


\section{CONCLUSIONS}

In summary, we demonstrate for the first time that probing of IR ISB polaritons in an isolated subwavelength size nanoantenna can be realized using scattering-type near-field FTIR spectroscopy. This is done through detection of evanescent fields on the nanoantenna surface. We demonstrate that the evanescent field exhibits a distinctive polariton anti-crossing behavior, where the nanoantenna resonance splits into two polariton branches if it is in resonance with the ISB excitation. Using the near-field approach, we mapped polariton dispersion in the single nanoantenna regime.

\section{ACKNOWLEDGEMENTS}

This work was supported by the U.S. Department of Energy, Office of Basic Energy Sciences, Division of Materials Sciences and Engineering. Fabrication and nano-FTIR measurements were performed at the Center for Integrated Nanotechnologies, an Office of Science User Facility operated for the U.S. Department of Energy (DOE) Office of Science. Sandia National Laboratories is a multi-mission laboratory managed and operated by National Technology and Engineering Solutions of Sandia, LLC., a wholly owned subsidiary of Honeywell International, Inc., for the U.S. Department of Energy's National Nuclear Security Administration under contract DE-NA-0003525. Los Alamos National Laboratory, an affirmative action equal opportunity employer, is managed by Triad National Security, LLC for the U.S. Department of Energy's NNSA, under contract 89233218CNA000001. This article describes objective technical results and analysis. The views expressed in the article do not necessarily represent the views of the U.S. DOE or the United States Government. C.-F. W. and T. H. were supported in part by the U.S. National Science Foundation (Grant No. 1651478).

\section{REFERENCES}

[1] Kockum, A. F., Miranowicz, A., De Liberato, S., Savasta, S. and Nori, F., "Ultrastrong coupling between light and matter," Nat. Rev. Phys. 1, 19 (2019).

[2] Colombelli, R. and Manceau, J.-M., "Perspectives for Intersubband Polariton Lasers," Phys. Rev X 5, 011031 (2015).

[3] Todorov, Y. and Sirtori, C., "Intersubband polaritons in the electrical dipole gauge," Phys. Rev. B 85, 045304 (2012).

[4] Benz, A. et al., "Strong coupling in the sub-wavelength limit using metamaterial nanocavities," Nat. Commun. 4, 2882 (2013).

[5] Malerba, M. et al., "Towards strong light-matter coupling at the single-resonator level with sub-wavelength midinfrared nano-antennas," Appl. Phys. Lett. 109, 021111 (2016).

[6] Khoromova, I. et al., "Splitting of magnetic dipole modes in anisotropic TiO2 micro-spheres," Laser and Photonics Reviews 10, 681 (2016).

[7] Mitrofanov, O. et al., "Detection of internal fields in double-metal terahertz resonators," Appl. Phys. Lett. 110, 061109 (2017).

[8] Mitrofanov, O. et al., "Near-field spectroscopy and tuning of sub-surface modes in plasmonic terahertz resonators," Opt. Express 26, 7437 (2018).

[9] Hale, L.L. et al., "Near-Field Terahertz Spectroscopy: Noninvasive Near-Field Spectroscopy of Single Subwavelength Complementary Resonators," Laser and Photonics Reviews 10, 1900254 (2020).

[10] Olmon, R.L. et al., "Near-field imaging of optical antenna modes in the mid-infrared," Opt. Express 16, 20295 (2008).

[11] Bohn, B.J. et al., "Near-Field Imaging of Phased Array Metasurfaces," Nano Lett. 15, 3851 (2015).

[12] Wang, C.-F. et al., "Observation of Intersubband Polaritons in a Single Nanoantenna Using Nano-FTIR Spectroscopy," Nano Lett. 19, 4620 (2019). 